\title{
Ambiguity as a Resource for Design
}

\author{
William W Gaver ${ }^{1}$ \\ ${ }^{1}$ Interaction Design Research Studio \\ The Royal College of Art \\ London, SW7 2EU, UK \\ w.gaver / j.beaver@rca.ac.uk
}

\author{
Jake Beaver $^{1}$
}

Steve Benford ${ }^{2}$

${ }^{2}$ The Mixed Reality Laboratory

University of Nottingham

Nottingham, NG8 1BB, UK

mbl@cs.nott.ac.uk

\begin{abstract}
Ambiguity is usually considered anathema in Human Computer Interaction. We argue, in contrast, that it is a resource for design that can be used to encourage close personal engagement with systems. We illustrate this with examples from contemporary arts and design practice, and distinguish three broad classes of ambiguity according to where they are located in the interpretative relationship linking person and artefact. Ambiguity of information finds its source in the artefact itself, ambiguity of context in the sociocultural discourses that are used to interpret it, and ambiguity of relationship in the interpretative and evaluative stance of the individual. For each of these categories, we describe tactics for emphasising ambiguity that may help designers and other practitioners understand and craft its use.
\end{abstract}

\section{Keywords}

Interaction design, Emotion and Affective UI, Design, Theory, Design Tips and Tricks, Heuristics, Interpretation

\section{INTRODUCTION}

Ambiguity is seldom embraced within traditional Human Computer Interaction. If "usefulness and usability are the twin goals of HCI research and development" [14], then ambiguity would seem their nemesis. It is difficult to see how an interface can be usable if the information it conveys is unclear, or useful if its very purpose is uncertain.

Instead of regarding ambiguity as a problem, however, in this paper we suggest that it can be seen as an opportunity. Ambiguity can be frustrating, to be sure. But it can also be intriguing, mysterious, and delightful. By impelling people to interpret situations for themselves, it encourages them to start grappling conceptually with systems and their contexts, and thus to establish deeper and more personal relations with the meanings offered by those systems.

The ability for ambiguity to evoke personal relationships with technologies is particularly relevant as digital technologies are designed to support activities outside of work. Traditional concerns for clarity and precision are

CHI 2003 submission superseded in such systems by the need to provide rich resources for experience that can be appropriated by users. Ambiguity should not, of course, be allowed interfere with the accomplishment of well-defined tasks, particularly in safety-critical environments. But in the many emerging applications for everyday life, we argue that ambiguity is a resource that designers should neither ignore nor repress.

After all, the everyday world itself is inherently ambiguous: most things in it have multiple possible meanings. Allowing this ambiguity to be reflected in design has several advantages. Most importantly, it allows designers to engage users with issues without constraining how they respond. In addition, it allows the designer's point of view to be expressed while enabling users of different sociocultural backgrounds to find their own interpretations. Finally, ambiguity can make a virtue out of technical limitations by providing the grounds for peoples' interpretations to supplement them.

The rich aesthetic and conceptual potentials of ambiguity have long been exploited in the arts, and we review several examples of contemporary art in considering how ambiguity works. But we are particularly concerned with examining how ambiguity can be used to good effect in design. To orient our discussion, we start by describing four examples of ambiguity in interaction design, which illustrate the appeal of ambiguity and some of its attributes. We then discuss an account of ambiguity that centres on its locus in the relationship between user and system, and show how this can help us understand ambiguity in a variety of situations. Finally, we return to the original four examples, as well as other designs, to illustrate a number of tactics for producing ambiguity. Our purpose throughout is to reveal some of the tricks that interactions designers use in creating compelling designs, and to enable other designers and practitioners to think more precisely - whether critically or constructively about ambiguity.

\section{Four Systems that Use Ambiguity}

Although ambiguity is usually avoided in Human Computer Interaction, there are a growing number of interactive designs that use it to good effect. In this section we describe four in order to provide concrete examples of how ambiguity can appear in technological systems, before moving to a more analytical account of how it operates. 


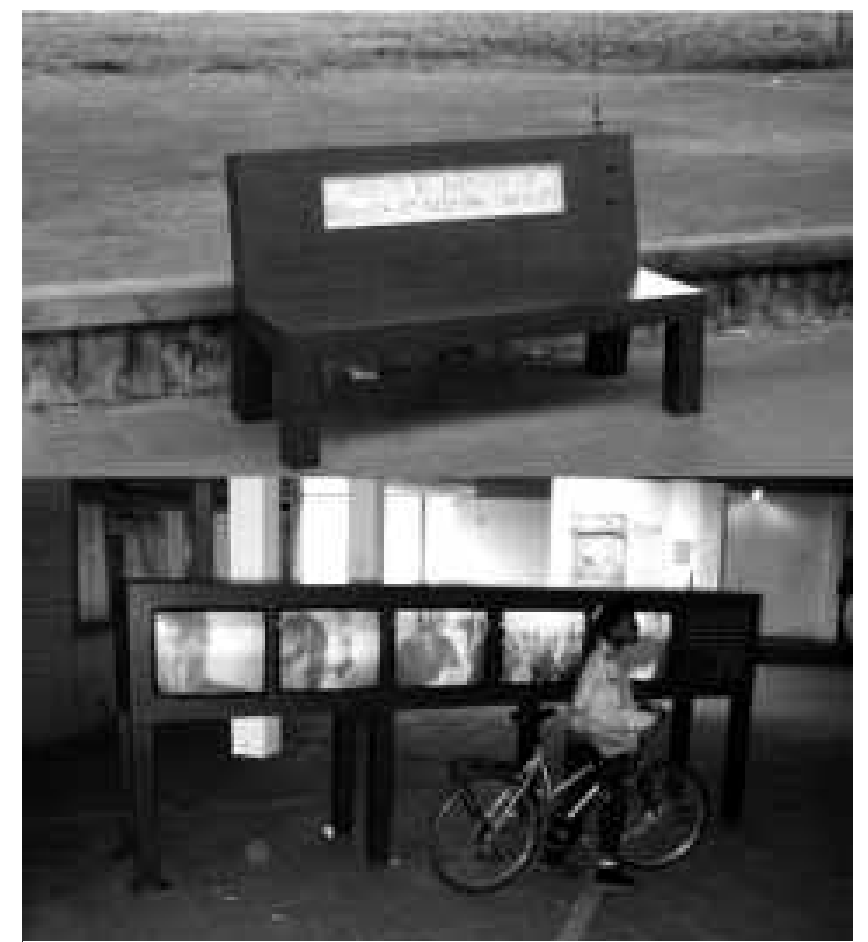

Figure 1: Sloganbench (top) and Imagebank (bottom)

\section{Projected Realities}

Projected Realities [8, 9] is a system intended to help increase the presence of older people in a large Dutch housing estate called the Bijlmer. Designed to reflect both concerns that older people felt about the area (which has a bad reputation in the Netherlands) and their pride for this complex physical and cultural environment, the system provided a means for them to spread their opinions within and to the outskirts of the district.

The most visible elements of the completed system were three Sloganbenches set in local walkways, and an Imagebank designed to sit near a traffic signal adjacent to the area (Figure 1). Sloganbenches served as public furniture, but also contained electronically-controlled fabric scrolls inset into their backs which displayed handwritten slogans written by older people from the area. The Imagebank comprised five monitors set into a wood-veneer casing. It showed slices of images collected by the older people to represent their lives. The pieces were connected wirelessly, with slogans appearing on the Sloganbench determining images that displayed on the Imagebank.

Projected Realities used ambiguity in a number of ways. The slogans and images were presented with little context (even the fact that they originated from older people was spread by word of mouth) so that viewers had to interpret the attitudes they reflected. The mapping between slogans and images was not easily perceived, and occasionally contradictory images were juxtaposed on the Imagebank. Most notably, the objects themselves were unusual - the Imagebank looked like an extended TV, and the benches created a tension between sitting and viewing (since sitting on the bench was likely to hide the slogan). They balanced the familiar with the strange, and as local people encountered the pieces during a week-long trial in the area, they were attracted by their ambiguity to engage with the system.

\section{Desert Rain}

Desert Rain is a mixed reality performance that has toured internationally since 1999 [12]. Six players (the audience) are sent on a mission into a virtual world to find six targets (the performers). They explore motels, deserts and underground bunkers, communicating with each other through a live audio link. Once in the virtual world, they have twenty minutes to find their allocated targets, complete the mission, and get to the final room, where the identities of the targets - six people who have quite different perspectives on the Gulf war - are revealed. The virtual world is projected onto six rain curtains, screens made of water through which performers and players physically pass (Figure 2). In turn, the rain curtains are embedded within a larger physical set, some of which reproduces elements of the virtual world.

The central artistic concern of Desert Rain is virtual warfare and the blurring of the boundaries between real and virtual events, especially with regard to the portrayal of warfare on television news, in Hollywood's films and in computer games. Both the content and the form of Desert Rain are designed to provoke participants to re-evaluate the boundaries between reality and fiction, and between the real and the virtual. This is achieved by making these boundaries deliberately ambiguous. Desert Rain mixes elements of theatre, installation and computer game to create an ambiguous structure. At the same time, its content mixes 3D game-like graphics with video clips describing people's real experiences of the Gulf War. It also seeks to disorientate participants to some extent by providing them with deliberately distorted and ambiguous information, most notably through the use of the rain curtain which provides a continually shifting and blurred view of the virtual world.

\section{The Pillow}

The Pillow, a concept prototype by Tony Dunne [8], employs ambiguity at several levels. An LCD screen embedded in a plastic brick is enclosed by a transparent plastic pillow, so that the simple geometric shapes it

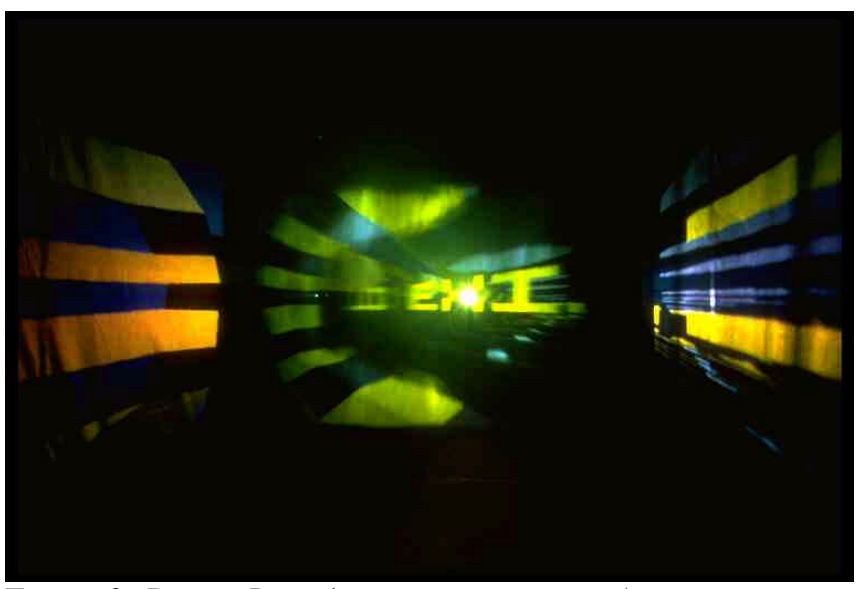

Figure 2: Desert Rain (projections on water) 


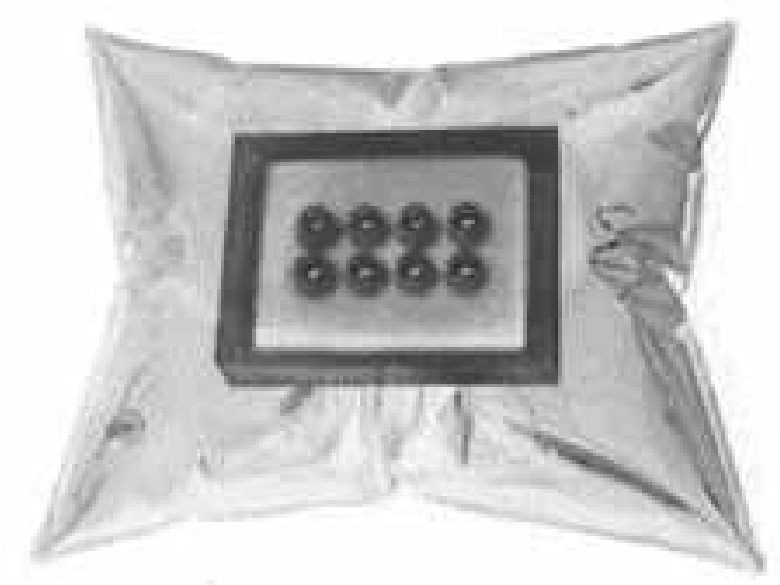

Figure 3: The Pillow

displays are blurred as they permeate the form (Figure 3 ). These shapes join with processed sounds to indicate passing electromagnetic information from mobile phones, taxis, commercial radio and television, making the Pillow into an unusual sort of radio - one that, moreover, casts the viewer into a voyeuristic relationship with the otherwise invisible information that surrounds us.

The Pillow displays electronic information in an impressionistic, ambiguous way. One can perceive the intensity of electromagnetic flow and capture snatches of intelligible sound, but the information is distorted and fragmented to produce an intriguing rather than informative experience. In addition, the Pillow exists at the cusp of several discourses: it is a radio, but also an aesthetic meditation - and perhaps also an artistic one. Finally, the Pillow produces yet another layer of ambiguity: by placing the user in a voyeuristic role it creates an ambivalent relationship with the purpose of the piece itself. The ability to eavesdrop on the electromagnetic spectrum is enticing yet uncomfortable, prompting questions about the ethics of technologies.

\section{Home Health Monitor}

The Home Health Monitor is a design for a system that gives feedback about the home's emotional, social and spiritual health on a daily basis [2]. Light, temperature, and door sensors, for example, are used to monitor peoples' activities in various parts of the home. Other sensors measure the stroke rate of a hairbrush, condensation on kitchen windows, or the state of the toilet seat to provide more idiosyncratic clues. Sensor readings and trends are mapped in state space to a collection of sentences drawn from a large number of published horoscopes to generate a bespoke horoscope for the particular household on a given day (Figure 4).

By using ubiquitous sensing as an indication of the wellbeing of the home, the system (sometimes known as 'the Superstitious Home') offers an alternative to traditionally task-oriented forms of ubiquitous computing. It achieves its effect by allowing horoscopes to provide an ambiguous reflection of the state of sensors, which themselves have an ambiguous relationship to the realities of home life. This ambiguity ranges from the limited ability of sensors to monitor home activities to the relationship between measurable variables and emotional ones, and from the vague language used by most horoscopes to the juxtaposition of electronic technology with culturally suspect 'fortune-telling.' The end result is to provide people with a systematic but inconclusive foundation from which to reflect on the emotional state of their home.

\section{Clarifying Ambiguity}

These examples illustrate the wide variety of systems that may benefit from ambiguity. They include products, systems and events, and their intended settings include homes, performance spaces, and public areas. Insofar as they are similar to one another, this is because their use of ambiguity makes them evocative rather than didactic and mysterious rather than obvious. They embody a style of interaction that, while relatively new to HCI, is potentially as encompassing as the more traditional style of clear practicality. This style hinges, in part, on their use of ambiguity, and they illustrate several of its features that are useful for its understanding.

Most importantly, they highlight the fact that ambiguity is a property of the interpretative relationship between people and artefacts. This distinguishes ambiguity from related concepts such as fuzziness or inconsistency: these are attributes of things, whereas ambiguity is an attribute of our interpretation of them. Things themselves are not inherently ambiguous. They may give rise to multiple interpretations depending on their precision, consistency, and accuracy on the one hand, and the identity, motivations, and expectations of an interpreter on the other.

This interpretative relationship is the source of ambiguity's appeal: by thwarting easy interpretation, ambiguous situations require people to participate in making meaning. This can involve the integration of previously disconnected discourses, the projection of meaning onto an unspecified situation, or the resolution of an ethical dilemma. In each case, the artefact or situation sets the scene for meaningmaking, but doesn't prescribe the result. Instead, the work of making an ambiguous situation comprehensible belongs to the person, and this can be both inherently pleasurable and lead to a deep conceptual appropriation of the artefact.

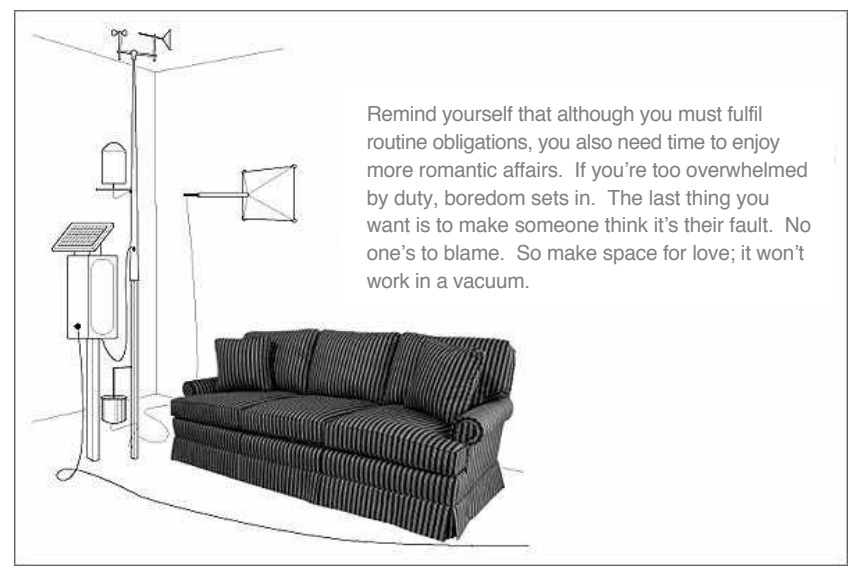

Figure 4: Home Health Monitor 


\section{TYPES OF AMBIGUITY}

Despite the fact that ambiguity is a property of the an interpretative relationship, the likelihood of an ambiguous interpretation can be affected either by attributes of artefacts or by people's attitudes alone. For example, interfaces that are imprecise or contradictory are more likely to lead to multiple interpretations than ones that are clear and consistent; people who expect clarity and consistency are more likely to perceive ambiguity than those who do not. In this paper, we focus on the first half of this interpretative relationship to consider how products and systems can be designed to elicit different kinds of ambiguity. In particular, we distinguish three principal kinds of ambiguity: ambiguity of information, of context, and of relationship.

\section{Ambiguity of Information}

Let's start by looking at an old master: Leonardo da Vinci's Mona Lisa (c. 1503-07). This is a straightforward figurative painting. Why is it so famous? As Charles Nicolls wrote:

We don't know precisely when it was painted, we don't know for certain who she is, and as we stare at her puzzling features for the umpteenth time we are inclined to ask ourselves: 'what is it about her?' [15]

All this ambiguity is centred on her smile. In Leonardo's notes on painting, he says that light and shade should blend 'without lines or borders, in the the manner of smoke'. [13] This technique is called sfumato, and it accounts for the mystery of Mona Lisa's smile. By reducing the definition (or focus) around her lips, Leonardo makes her whole expression indeterminate, and thus a 'terrain for infinite variations' [17]. With insufficient information to go on, the viewer has to bring that smile into focus in their mind.

Now consider an equally famous, modernist painting. Picasso's Guernica (1936) seems to work in the opposite way, by increasing the definition. The painting is full of strong 'lines and borders', rather like the figures in a cartoon strip. Yet this heightening of contrasts also results in loss of information, which produces a different kind of ambiguity. Are we to take these figures as portraits of unique individuals and animals, or as representations of generic types - that is to say, of ideas? And if each represents an idea, who's to say exactly which idea? The more one simplifies an image, the more iconic it becomes, and the more open to interpretation. As Read [16] points out, 'Does the bull represent the concept of violence, or the dictator Franco?' Again, the viewer has to decide. In order to make the image coherent, the viewer has to find a conceptual focus, as it were, which harmonises all the elements.

In both these examples, ambiguity arises in the way that information is presented. Now consider the more complex example of an interactive system:

Bystander is a prototype mixed reality game designed by one of the authors in collaboration with Blast Theory, a London performance arts group. In the game, two players, one online and the other equipped with mobile technologies, follow somebody moving through the city.
Both have access to limited and different kinds of information. In particular, the mobile player's location is tracked using GPS data, which is of low spatial and temporal resolution and also prone to errors. Thus the issue is not 'What does this display mean?' but 'Do I trust it?' - that is, how does the display correspond with reality?

The traditional response to ambiguity of information in interactive systems like Bystander is to improve the technology, use statistical methods to set certainty thresholds, or ignore it and hope for the best. Bystander, in contrast, passes the ambiguity directly to players in the form of fuzzy avatars that hint at locations without specifying them. Rather than seeing uncertain GPS information (in this instance) as a flaw, Bystander treats this ambiguous information as a challenge to users, forcing them to join their knowledge of people and cities to the clues offered by the system to play the game.

\section{Ambiguity of Context}

Ambiguity sometimes arises not because things are themselves unclear, but because they may be understood in different contexts, each suggesting different meanings.

One of the most notorious examples of this is Duchamp's Fountain, rejected from a 1917 exhibition (despite the organiser's guarantee to show two pieces for the fee of \$6) because it was a urinal turned on its side [11]. The Fountain is deeply controversial because it lends itself to several interpretations simultaneously. On the one hand, it is art: the work of one of the 20th century's most celebrated artists, sold recently for more than one million dollars [3], and shown in internationally respected museums. On the other hand, it is a just a commodity: a mass manufactured 'ready made,' not created by Duchamp at all, and, to add insult to injury, a toilet.

To view the Fountain - or just to think about it - is to enter into this deeply ambiguous situation. Seen as a sculpture, the Fountain's graceful lines and intimate enclosure can be aesthetically pleasing. But it is, almost insistently, a urinal. Not only can it be perceived in different ways, but for many people these interpretations are incompatible. Attempts to resolve this tension can be absurd or violent, as when in 1993 a painter first urinated in the Fountain and then hit it several times with a hammer [3]. But this ambiguity and the tensions it evokes are the point of the piece: the source of its power and the conceptual pleasure it has to offer.

It is not unusual to find artworks designed to be ambiguous. It is more difficult, in contrast, to find examples of commercial products that are ambiguous in terms of their preferred context of interpretation. Product designers work to eliminate ambiguity: their main effort goes into balancing clarity of use (making it intuitive) with richness of semiotic suggestion (making you like what it stands for). Both aspects of the design attempt to control the user's interpretation of the product - that is, to reduce ambiguity.

However, now that technology is no longer used solely by specialists, with specialist requirements, its users are 
becoming ever more varied and its uses ever more flexible. Despite the best efforts of product designers, products and services are increasingly treated as if they were open to interpretation. Users will even go against the (semiotic) grain in order to reinterpret a product. Sometimes these reinterpretations will go beyond new applications of an understood product to redefine the very purpose of the thing itself, creating a contextual ambiguity around it.

For example, the abundance of different ring tones on mobile phones is used by advertisers as an incentive to the youth market. But some mothers have now started playing with ring tones - not to be cool, but to soothe their babies. The mother sets the phone ringing and hands it to the wailing infant. Note that this does not simply involve an unexpected use of the phone to communicate. Instead, much as Duchamp transmuted a base urinal into a celebrated piece of art, so the phone has been transformed into a new kind of baby rattle. This reveals the ability to interpret mobile phones in terms of discourses alien to those of their designers - a possibility that designers can recognise and encourage.

Beyond noting and encouraging novel reconceptualisations of existing technologies, however, designers can deliberately set out to create products that implicate several interpretations at once. When successful, this mingling of discourses disrupts easy interpretation of the design, and obliges users to work out ways to make sense of the new situation.

\section{Ambiguity of Relationship}

As an example of a third type of ambiguity, consider Van Lieshout's Bais-ô-Drôme [1]. This is a 'love caravan' consisting of two molded polyester enclosures that are fitted together like an extended trailer. The nondescript and even foreboding exterior hides an interior that might be described as 'functionally decadent.' Liquor bottles are hung on the walls for easy access from a large, cushioned settee, while in the background a sheepskin-covered platform seems simultaneously clinical and sleazy. The whole is a well-constructed and integrated design that manages to leave viewers admiring but uncomfortable.

There is nothing particularly unclear about the presentation of this piece. In addition, though it extends and merges known product genres in interesting ways, these seem to coexist without tension (though the Duchampian move of claiming a living space as art may trouble some viewers). The ambiguity, instead, arises from viewer's personal relationship with the piece. It is admirably well-crafted but would you want to inhabit it? Under what circumstances? And should so much effort and care be devoted to such a strange mixture of utility and debauchery?

Gaver and Martin [10] developed design proposals intended to produce a similar sort of self-examination. The Prayer Device, for example, transmits users' voices straight up to the skies, allowing people to send their thoughts or supplications to whomever or whatever they think might listen. The Telegotchi is an electronic pet with no buttons, relying on psionic powers for influence. Both these designs work within understood contexts - of spirituality on the one hand, and telepathy on the other - and invite users to share the experience of believers.

Designs like these do not simply engender ambivalence, or contradictory emotion. They push us to imagine how we might personally use such products, and what our lives would be like in consequence. As a result of this speculation, we form intellectual, aesthetic, emotional, and moral judgements that can become available for selfreflection. The result of this process can be experiences that are uniquely personal: delightful, disturbing, or both.

In summary, the three kinds of ambiguity raise different sorts of problem and ask for different sorts of interpretation. Ambiguity of information asks us to project our expectations into an interpretation of incomplete information. Ambiguity of context requires an integration of seemingly incompatible meanings. Ambiguity of relationship, finally, evokes a projection of our subjective experience at many levels.

\section{TACTICS FOR USING AMBIGUITY}

We chose the examples in the section above both to illustrate how ambiguity is used in the arts and design, and because they are relatively clear illustrations of the three primary loci of ambiguity. In this section, we discuss each kind of ambiguity in more detail, describing specific heuristics for achieving them with reference to our original design examples as well as new ones. Note that our intention here is not to present an exhaustive or exclusive analysis (indeed we question whether such an analysis would be practical or desirable). Instead, we articulate these tactics to help designers recognise, understand and use ambiguity.

\section{Enhancing Ambiguity of Information}

A number of tactics may be used to enhance ambiguity of information. These focus on creating or reflecting uncertainties about information that are noticeable to people. The purpose of this may be merely to make the system seem mysterious or impressionistic, but more importantly it can also compel people to join in the work of making sense of a system and its context.

\section{Use imprecise representations to emphasise uncertainty.}

Perhaps the most prevalent way to introduce ambiguity of information is to display information that is physically or conceptually blurred. For instance, Desert Rain exploits ambiguity of information through the use of the rain curtain to create blurred and shifting images of the virtual world. This treatment is similar to Dunne's use of a translucent plastic brick and pillow to obscure the graphics on an LCD display. In both cases, a kind of digital sfumato is achieved by blurring the usually precise quality of digital displays.

The use of deliberate imprecision can extend to tangible and textual displays as well. For instance, Jeremijenko's LiveWire [18] used a spinning cable to indicate ethernet traffic, while Ishii [5] used pinwheels to indicate network load. The Home Health Monitor appropriates the tendency of horoscopes to use diectic wordings that make vague 
statements seem definite (e.g. "work to maintain the creative areas that are personally rewarding and cut your losses elsewhere"). The Sloganbenches used short, decontextualised phrases to create a similar air of indefiniteness (e.g. "I used to be restless because of negative thoughts")

Imprecise displays such as these are often described as 'ambient', but they are ambiguous as well. This ambiguity, we believe, is crucial for understanding their appeal: they may be perceptually undemanding, but they require users to fill in the gaps in information that is purposefully imprecise. When successful, such interfaces are not only aesthetically attractive, but conceptually appealing as well.

Over-interpret data to encourage speculation.

The converse of using blurred representations of information is to over-interpret them (in much the same way that Guernica's heightened definition contrasts with the indistinct features of the Mona Lisa). For instance, the Home Health Monitor uses over-interpretation in making statements about the emotional state of the home based on the input from physical sensors. The apparent overconfidence of the system raises questions about the degree to which such interpretations might be justified, seducing users into considering the well-being of their home through their scepticism about the truth of the system's suggestions.

Over-interpretations should not be clear nonsense, however. An earlier proposal for a Tweet-to-Text system using voice recognition to give pets the chance to speak [2] is questionable in this regard: it is difficult to suspend disbelief and entertain the notion that the results might be sensible. The design equivalent of gross exaggeration (e.g. "I've been working on this paper for a million years"), over-interpretation is best used to draw attention to possible truths rather than simple untruths.

\section{Expose inconsistencies to create a space of interpretation.}

Inconsistent information from interfaces can also encourage interpretative engagement. For example, the Imagebank used in the Projected Realities system sometimes showed multiple, inconsistent images (e.g. a quiet family scene next to a protest march) to reflect inconsistent slogans appearing on the Sloganbenches. This had the effect of opening a space of possible interpretations - the juxtaposed images could be read as indicating the range of Bijlmer realities, or as a mutual rebuke, or as a narrative. However it was interpreted, the point is that it was interpreted, that the juxtaposition of incompatible elements required viewers to build their own meanings from the display.

Exposing inconsistency between sources of information (e.g. external sensors) can also be a powerful alternative to trying to resolve or hide it. For instance, just as the uncertainties of GPS tracking in Bystander are shown to participants, so are inconsistencies revealed between GPS and spoken information. By crafting the inherent limitations of the technology into the user experience, they become a means to heighten narrative intrigue by opening a space of possibilities that participants must navigate.
Cast doubt on sources to provoke independent assessment.

Over-interpretation and inconsistency are special cases of increasing ambiguity by casting doubt on sources of information. For instance, not only are the Home Health 'horoscopes' imprecise, but they are produced by an unseen mapping from a pattern of sensor readings which themselves have questionable relation to the statements being made. The result is to force users to decide for themselves the credence to put on the predictions, either by tracing back the chain of cause and effect in the system or more simply by gauging whether the statements match their experiences.

Similarly, because the slogans displayed by the Sloganbenches were not contextualised it was difficult on first viewing to recognise that they were produced by older people (this information spread by word of mouth as the system was used). Instead, they appeared simply as statements to be believed or disbelieved. In this they are similar to the artist Jenny Holzer's Truisms (1977-9), electronic displays that show a series of bald assertions (e.g., 'money creates taste') that viewers can accept or question. The statements shown by the Sloganbenches, however, could be traced back to the older people, and this tension between abstraction and individuality allowed people to gauge the statements per se or as expressions of older people.

\section{Creating Ambiguity of Context}

Blocking the interpretation of a product or system in terms of an established discourse can create ambiguity of context. This is useful in spurring people to approach a particular system with an open mind, and more generally to question the assumptions they have about technological genres.

\section{Implicate incompatible contexts to disrupt preconceptions.}

Though none of our introductory design examples implicate incompatible contexts as powerfully as Duchamp's Fountain, all use this tactic to some degree in interrupting an easy interpretation. The Sloganbenches are not quite public furniture or public displays, and treating them simply as one or the other is problematic (sitting on them blocks the displays; viewing the displays blocks sitting). The Pillow is simultaneously a radio and an aesthetic object. Desert Rain juxtaposes the established media forms of theatre, interactive installation and computer games, and moreover plays like a computer game despite being an anti-war piece.

In each case, bringing together disparate contexts creates a tension that must be resolved. To engage with the design, participants must rethink their basic assumptions about genre, and either privilege one discourse over the others or expand their understandings of the discourses to build bridges among them. In doing so, they are encouraged to face the system - and perhaps even technological genres in general - from a fresh perspective.

\section{Add incongruous functions to breach existing genres.}

Adding new functions to designs can sometimes move them out of their 'home' genres. From this perspective, the conceptual origin of the Sloganbenches as public 
furniture is apparent, but they become something different through the addition of the scrolling displays. Accommodating this augmentation to the notion of public furniture is possible, but requires a redefinition that is fundamental enough to be uncomfortable.

Similarly, products can split from their original genres when their functionality is used in radically new contexts, as for instance in our example mobile phones being used as baby rattles. For instance, The Sailor's Return is a design proposal for a man who splits his time between an apartment and a two-masted sailing ship [2]. It suggests that his coffee cup could be linked wirelessly to a neon sign (picturing a coffee cup, of course) outside his apartment window. Returning from the ship and having a cup of coffee would cause the sign to light up, signalling to his friends and neighbours that he is home from the sea. Apart from casting the coffee cup as an activity monitor (a mixing of genres that is common in ubiquitous computing research), this proposal transforms a public sign into a private code, allowing us to rethink the potential of public displays in new ways.

\section{Block expected functionality to comment on familiar products.}

Taking away functionality can also separate designs from their native genres. This is yet another way to understand the effect of being able to obscure the Sloganbench displays by sitting on them. Another example of this tactic is a design proposal called Democratic Advertising [10], in which people could use handheld devices to capture and redistribute advertisements shown on public displays. This can be seen as a political device, allowing people unprecedented influence over the visual appearance of public spaces. Yet according to this proposal, people can only move existing advertisements, they cannot alter them or create their own imagery. This can seem frustrating, but it adds an intriguing contextual ambiguity. The system is not a straightforward tool for advertising, nor is it just a simple utopian proposal for legal graffiti; instead it finds a more delicate balance between the two.

Perhaps the purest example of blocking functionality to achieve ambiguity of context is Sarah Pennington's design of a mobile phone cover that has no 'call' or 'receive' buttons. The phone is transmuted into a device that can only signal when somebody is trying to call: the owner can't pick up or make calls. Through this simple modification, Pennington disrupts our reading of the phone as a communications device, creating instead a rather plaintive reminder of separation. But the result doesn't escape its origin as a phone: it is simultaneously phone and not-phone, and this ambiguity is central to its effect.

\section{Provoking Ambiguity of Relationship}

Ambiguity of relationship creates the condition for a deeply personal projection of imagination and values onto a design. This can allow products and systems to become psychological mirrors for people, allowing them to try on new identities or to question their values and activities.

\section{Offer unaccustomed roles to encourage imagination.}

By their aesthetics and the functionality they offer, products and systems imply a 'narrative of use' [6] and suggest the sorts of people for whom they are appropriate. Designers traditionally strive to craft the semiotics of their products so that a majority of people will relate or aspire to the identities they imply. Reflecting more idiosyncratic roles and activities, however, can give rise to products that create an ambiguity of relationship, spurring people to question their own values.

Dunne's Pillow [7] is a seminal example of applying ambiguity of relationship to electronic products. Culling electromagnetic information from sources that might include mobile phone calls or baby monitors, it entices people to take a voyeuristic role to the airwaves. Similarly, the Prayer Device and Telegotchi also encourage people to adopt potentially unfamiliar perspectives and activities. Described by Dunne [6] as 'value fictions,' these sorts of design use possible technologies to explore implausible values. They act as props for a drama played in everyday life, allowing people to engage in a first-person narrative as a kind of experiment in living.

Point out things without explaining why.

Offering new roles to people emphasises issues of personal identity. Another form of ambiguity of relationship, however, encourages people to consider the personal significance of things, behaviours, or events in their environment. Much as fine art photography has shifted from capturing images of important scenes to making scenes important by capturing their image, so design can draw attention to overlooked aspects of the environment to encourage reflection on their significance.

For example, the rationale for tracking certain physical events for the Home Health Monitor is non-obvious, but the mere fact of their being tracked may arouse curiosity among participants. The flip side of asking whether open doors and frequently-used hairbrushes really reflect wellbeing is to ask what these things do mean. In a similar way, the History Tablecloth [2] uses load sensors and electroluminescent film printed on fabric to highlight locations of objects that have been left on household surfaces for days or weeks. Based in part on ethnographic observations of how people use household surfaces [4], the cloth encourages people to reflect on the different histories of objects in their environment. But their interpretation is left open: the Tablecloth purposefully allows the meaning of its display to remain ambiguous to its users. In doing so, it encourages them to reflect on the meaning of this aspect of their environment, aesthetically, culturally, and especially - personally.

Introduce disturbing side effects to question responsibility.

Reflection about the balance of desire and ethics can be provoked by designs that seem immediately appealing but which have disquieting implications. The Pillow works this way in providing an aesthetic experience that only slowly reveals itself to depend on eavesdropping: it seduces people into a voyeuristic role that may be uncomfortable. As another example, the Dawn Chorus is a proposal for an 
artificially-intelligent feeder that uses operant conditioning principles to teach local birds new songs [10]. This whimsical design may be appealing, but on reflection it raises disturbing issues about the ecological effects of interfering with birds' natural behaviour, not to mention the ability to broadcast one's tastes (what if my neighbour is a Britney Spears fan?).

Designs such as these reflect and amplify the difficult choices posed by many current technologies. Mobile phones, for instance, allow us to communicate freely, but at the cost of intruding on others and of losing our solitude. Reactive environments may offer convenience at the cost of privacy. Portable computers interfere with the separation of work and home. Such tradeoffs are endemic to new technologies; proposals such as the Pillow and Dawn Chorus provoke us to reflect on these tradeoffs both in the particular and more generally.

\section{CONCLUSION: AMBIGUITY AS A VIRTUE}

Ambiguity is not a virtue for its own sake, nor should it be used as an excuse for poor design. Many ambiguous systems are merely confusing, frustrating, or meaningless. Nonetheless, as we hope to have shown in this paper it can be an important factor in crafting interactive designs that are engaging and thought-provoking. Moreover, it has the added advantage (which we have purposefully avoided stressing in this paper) of enabling designers to go beyond the limits of their technologies. From this point of view, ambiguity provides a context that allows the use of inaccurate sensors, inexact mappings, and low-resolution displays because it encourages users to supplement them with their own interpretations and beliefs.

The most important benefit of ambiguity, however, is the ability it gives designers to suggest issues and perspectives for consideration without imposing solutions. Ambiguity of information impels people to question for themselves the truth of a situation. Contextual ambiguity can question the discourses surrounding technological genres, allowing people to expand, bridge, or reject them as we see fit. Relational ambiguity, finally, can lead people to consider new beliefs and values, and ultimately their own attitudes. In each of these cases, ambiguity frees users to react to designs with scepticism or belief, appropriating systems into their own lives through their interpretations. In the process of reacting to the system either positively or negatively, however, users engage with issues that the designer suggests. Thus ambiguity is a powerful tool for designers to raise topics or ask questions while renouncing the possibility of dictating their answers. By supporting this balance, ambiguity not only represents a useful resource, but a powerful sign of respect for users as well.

\section{REFERENCES}

1. Atelier van Lieshout. A manual. Kölnischer Kunstverein and Museum Boijmans van Beuningen, Cologne, 1997.

2. Boucher, A., Gaver, W., Pennington, S., and Walker, B. Equator workbook one: Ideas, scenarios and proposals for the home. Royal College of Art, 2001.

3. BBC online. Available at: http://news.bbc.co.uk/1/hi/ entertainment/arts/1987109.stm

4. Crabtree, A., Hemmings, T., and Rodden, T. Pattern-based support for interactive design in domestic settings. Proc. DIS2002 (London 2002). ACM Press, pp. 265 - 275.

5. Dahley, A., Wisneski, C., and Ishii, H. Water Lamp and Pinwheels: Ambient projection of digital information into architectural space. Proc. CHI'98 Companion (1998), ACM Press, pp. 269-270

6. Dunne, A. Hertzian tales: Electronic products, aesthetic experience and critical design. London, RCACRD Research Publications, 1999.

7. Dunne, A., and Gaver, W. The Pillow: Artist-designers in the digital age. Proc. of CHI'97 Companion (Atlanta, 1997), ACM Press.

8. Gaver, W., and Dunne, A. (1999). Projected Realities: Conceptual design for cultural effect. Proc. of $\mathrm{CHI}^{\prime} 99$ (1999), ACM Press.

9. Gaver, W., and Hooker, B. The Presence Project. London, RCACRD Research Publications, 2001.

10. Gaver, W., and Martin, H. Alternatives: Exploring information appliances through conceptual design proposals. Proc. of CHI'00 (Den Haag). New York, ACM Press.

11. Godfrey, T. Conceptual Art. London, Phaidon, 1998.

12. Koleva, B., Taylor, I., Benford, S., Fraser, M., Greenhalgh, C., Schndelbach, H., vom Lehn, D., Heath, C., Row-Farr, J. \& Adams, M. Orchestrating a Mixed Reality Performance. Proc. CHI'01, (Seattle, 2001), ACM Press.

13. Murray, P., and Murray, L. The Penguin Dictionary of Art and Artists. London: Penguin Books, 1983.

14. Nickerson, R. \& Landauer, T. Human-computer interaction: Background and issues. In Helander, M.G., Landauer, T.K. and Prabhu, P. (eds.), Handbook of HumanComputer Interaction, 2nd edition. Amsterdam, The Netherlands: Elsevier Science.

15. Nicolls, C. The Mona Lisa, London Review of Books, 4 April 2002.

16. Read, H. A Concise History of Painting. London, Thames \& Hudson, 1959.

17. Sassoon, D. Mona Lisa: The History of the World's Most Famous Painting. London, HarperCollins, 2001

18. Weiser, M. and Brown J.S. Designing calm technology. PowerGrid Journal, v.1.01, July 1996. 